\title{
DEVELOPING GENE-SPECIFIC META-PREDICTOR OF VARIANT PATHOGENICITY
}

Anna Rychkova ${ }^{1}$, MyMy C. Buu ${ }^{2}$, Curt Scharfe ${ }^{3}$, Martina I. Lefterova ${ }^{4,5}$, Justin I. Odegaard ${ }^{4}$, Iris

$$
\text { Schrijver }{ }^{2,4}, \text { Carlos Milla }^{2}, \text { Carlos D. Bustamante }{ }^{1^{*}}
$$

${ }^{1}$ Department of Genetics, Stanford University School of Medicine, Stanford, CA 94305, USA

${ }^{2}$ Department of Pediatrics, Stanford University School of Medicine, Stanford, CA 94305, USA

${ }^{3}$ Department of Genetics, Yale University School of Medicine, New Haven, CT 06519, USA

${ }^{4}$ Department of Pathology, Stanford University School of Medicine, Stanford, CA 94305, USA

${ }^{5}$ Personalis, Inc., Menlo Park, CA 94025, USA

*Correspondence: cdbustam@stanford.edu

\section{$\underline{\text { Abstract }}$}

Rapid, accurate, and inexpensive genome sequencing promises to transform medical care. However, a critical hurdle to enabling personalized genomic medicine is predicting the functional impact of novel genomic variation. Various methods of missense variants pathogenicity prediction have been developed by now. Here we present a new strategy for developing a pathogenicity predictor of improved accuracy by applying and training a supervised machine learning model in a gene-specific manner. Our meta-predictor combines outputs of various existing predictors, supplements them with an extended set of stability and structural features of the protein, as well as its physicochemical properties, and adds information about allele frequency from various datasets. We used such a supervised genespecific meta-predictor approach to train the model on the CFTR gene, and to predict pathogenicity of about 1,000 variants of unknown significance that we collected from various publicly available and internal resources. Our CFTR-specific meta-predictor based on the Random Forest model performs better than other machine learning algorithms that we tested, and also outperforms other available tools, such as CADD, MutPred, SIFT, and PolyPhen-2. Our predicted pathogenicity probability correlates well with clinical measures of Cystic Fibrosis patients and experimental functional measures of mutated CFTR proteins. Training the model on one gene, in contrast to taking a genome wide approach, allows taking into account structural features specific for a particular protein, thus increasing the overall accuracy of the predictor. Collecting data from several separate resources, on the other hand, allows to accumulate allele frequency information, estimated as the most important feature by our approach, for a larger set of variants. Finally, our predictor will be hosted on the ClinGen Consortium database to make it available to CF researchers and to serve as a feasibility pilot study for other Mendelian diseases. 


\section{Introduction}

The advent of next-generation sequencing that is quick, accurate, and affordable has promised to usher in a new era of genomic medicine. However, a critical issue facing the development of sequencing-based tests is the interpretation of novel genetic variants in terms of their probability of causing disease. This is a particularly pressing problem with so-called "clinically relevant genes", including the cystic fibrosis transmembrane conductance regulator (CFTR) gene, for which DNA changes are known to impact phenotype, but for which the map of how each genotype affects the clinical phenotype is incomplete. Differentiating "benign" from "pathogenic" genetic variants is challenging, and often physicians are left with the unsatisfying and inconclusive result that their patient carries a "Variant of Unknown Significance" (VUS).

Despite recent advances in applying machine learning techniques to problems in biomedicine, existing computational approaches to variant classification all suffer from low overall accuracy rates. For example, SIFT ${ }^{1}$ and PolyPhen- $2^{2}$ are among the most widely used algorithms, but each has an accuracy of less than $70 \%{ }^{3}$. Their poor performance limits the clinical utility of these tools in determining whether a novel genetic variant is actually related to the disease of interest. We aimed to improve the performance of computational interpretation tools by developing a gene-specific metapredictor, focusing on the CFTR gene, which combines information from the most promising available tools supplemented with protein structure and stability features, physicochemical properties of mutated residues, and allele frequency information.

To develop this computational model, we focused our analyses on variants in the coding region of the CFTR gene. Despite recent progress in both sequencing and analysis techniques, interpreting the functional effect of variants in non-coding regions remains problematic due to insufficient training data. Therefore, to maximize the prediction capability of our model, we are initially focusing solely on the variants that are most likely to be relevant in terms of disease association, due to their relatively clearer relationship to protein structure.

Many bioinformatics methods have been developed for predicting the effect of missense mutations, which vary by the number of features included and the type of machine learning algorithm employed. The most advanced tools typically rely on amino acid sequence, protein structure, and evolutionary conservation for their prediction. For example, while SIFT relies solely on conservation, measured via multiple sequence alignment, PolyPhen-2 includes both sequence and structure-based features for prediction. The structure-based features in this context are used to describe the physical 
environment of the mutation, and include predictors such as solvent-accessible surface area, hydrophobic propensity, and the "mobility" of the atom. Another missense pathogenicity predictor, MutPred $^{4}$, uses a much larger set of structural parameters, including secondary structure, stability and intrinsic disorder, transmembrane and coiled-coil structure. In addition, MutPred utilizes functional properties of the protein, such as sites of post-translational modification, catalytic and DNA-binding residues. MutPred outperforms SIFT by $7 \%$ in the area under the ROC curve (AUC), and, more importantly, in addition to pathogenicity score can provide information about the molecular basis of the disease.

While SIFT, PolyPhen-2 and MutPred are trained using data from across the genome, genespecific pathogenicity prediction methods have also been developed. For example, Masica et al. ${ }^{5}$ created a CFTR-specific prediction algorithm called Phenotype-Optimized Sequence Ensemble (POSE). In contrast to methods utilizing multiple sequence alignment, POSE tries to iteratively construct an optimized sequence ensemble based on the performance of the scoring function, which uniquely integrates evolutionary conservation with physicochemical properties of the amino acids (such as charge, presence of aromatic or aliphatic group, hydrogen bond donor or acceptor, and signals for glycine and proline residues). POSE achieves a performance of $84 \%$, as measured by AUC on a training set of 103 CFTR variants, and importantly, the method displayed improved specificity when compared to tools trained genome-wide, implying a higher accuracy potential for methods trained on single genes.

Combining existing methods into a single predictor has proven to yield increased accuracy ${ }^{6,7}$. Successful examples of such meta-predictors, therefore, suggest that the separate methods used for prediction of variant-disease associations are orthogonal, and represent different biologically relevant relationships. The advantage of the machine learning classifier is its ability to integrate these orthogonal measures to identify predictive signatures of pathogenicity. Thus we are employing such a combination strategy in developing our CFTR-specific meta-predictor.

In addition to combining outputs from several existing prediction tools, we are also adding other useful features into out meta-predictor. Importantly, we integrate protein stability measures into our pathogenicity predictor. Protein stability is a fundamental property that affects function, activity, and regulation of biomolecules. Conformational changes are required for many proteins' function, implying that conformational flexibility and rigidity must be finely balanced. Incorrect folding and decreased stability are two of the major consequences of missense mutations, which can lead to disease. Protein stability is measured by the folding free energy change upon mutation, which is calculated as the 
difference in free energy between the folded and unfolded protein states ${ }^{8}$. Therefore, the estimated folding free energy change for each variant should give valuable information about the functional consequence of missense mutations.

By restricting our method to one particular gene, we are trying to take advantage of the protein structure information and extract from it features uniquely relevant for the CFTR protein. Unfortunately, the full protein structure for CFTR has not yet been solved with X-ray crystallography. However, a few homology models have been built based on the available crystal structure of the nucleotide-binding domain and the homologous ABC transporter, Sav1866 9,10 . Structural parameters that have been tightly fitted to the CFTR protein, as well as inferred changes in physicochemical properties induced by amino acid substitution, are valuable features that help to increase the overall method performance.

\section{$\underline{\text { Materials and Methods }}$}

\section{Variants data collection}

We utilized various data sources, both publically available and internal, to collect known protein coding variants in the CFTR gene (Table 1). Public data sources include The Clinical and Functional TRanslation of CFTR (CFTR2) ${ }^{11}$, the database of Genotypes and Phenotypes $(\mathrm{dbGaP})^{12}$, and the Exome Aggregation Consortium (ExAC) ${ }^{13}$. Internal resources include datasets obtained from the Stanford Cystic Fibrosis Center ${ }^{14}$, and the Stanford Molecular Pathology Laboratory ${ }^{15,16}$. In addition we also included variants used for training and testing the previously described POSE method, which was trained directly on $C F T R^{5}$. Overall, 1,899 protein coding CFTR variants have been collected, of which the majority (>60\%) are missense variants (Fig. 1A). Clinical significance (pathogenic, benign, variant of unknown significance (VUS)) was merged from different sources, and conflicting entries (reported as pathogenic by one source and benign by other) were considered as VUSs. Since the ExAC database does not report variants' pathogenicity, all the CFTR variants from ExAC were considered as VUSs. As expected, only a small portion of collected variants had pathogenicity evidence (14\% pathogenic, $7 \%$ benign) (Fig. 1B), with the majority ( $\sim 80 \%)$ having unknown significance.

The full dataset of 1,899 CFTR variants with clinical significance from corresponding sources can be found on GitHub (https://github.com/rychkova/CFTR-MetaPred). 


\section{Variants annotation}

Our meta-predictor is built by combining outputs from a number of the available prediction tools and supplementing them with information extracted from protein structure and allele frequency (Table 2). From the variety of pathogenicity prediction tools available we considered those based on evolutionary conservation only (PROVEAN ${ }^{17}, \mathrm{SIFT}^{1}, \mathrm{PANTHER}^{18}$ ), and those based on some additional structural information as well (PolyPhen-2 $2^{2}$, MutPred ${ }^{4}, \mathrm{CADD}^{19}$, POSE ${ }^{5}$ ). Information regarding individual allele counts and overall sample sizes of the different studies was combined and converted to allele frequencies of variants. We used density function in $\mathrm{R}$ with the default Gaussian smoothing kernel to estimate the probability density function from the allele frequencies. Given the importance of protein stability for proper cellular function, we also incorporated predictors of folding free energy change into our model $\left(\right.$ Eris $^{20}, \mathrm{PoPMuSiC}^{21}, \mathrm{FoldX}^{22}$ ). We used two available homology models of CFTR protein ${ }^{9,10}$ for each of the stability predictor, which gave rise to six total stability features.

We further created several structural parameters based on the information in UniProt ${ }^{23}$ and $3 \mathrm{D}$ CFTR protein structure, such as protein domains (extracellular loops, nucleotide binding domain 1 or 2 (NBD1 or NBD2), transmembrane domain 1 or 2 (TMD1 or TMD2), R domain), nucleotide binding residues, topology (cytoplasmic, transmembrane, or extracellular protein parts), regions of posttranslational modification (phosphorylation, glycosylation, palmitoylation, or ubiquitination sites), and involvement in protein-protein interaction (PPI_score). Our PPI_score for each residue is based on the number of times each residue is present in the motifs known to be important for protein-protein interaction and CFTR regulation. Information about protein-protein interaction motifs known for CFTR is based on the literature, and summarized in Table S1. On top of these we added information about membrane contacting residues by building a simplified membrane model around the protein (using Coarse Grained model building tool in Molaris ${ }^{24}$ ), and selecting neighboring to membrane atoms residues in $\mathrm{PyMol}^{25}$. Similarly, we created a feature with channel contacting residues, by inserting a straight helix into the channel and selecting neighboring residues in PyMol.

We used DSSP tool ${ }^{26}$ to calculate change in solvent accessible area and hydrogen bond energy of the full protein as well as single residue upon mutation. Structural models of all the 1,210 mutant proteins (missense variants plus initiator codon variants) were obtained with Eris program ${ }^{20}$ using the default fixed-backbone method. Change in several physicochemical properties of residue due to 
mutation was estimated based on the information in the AAindex dataset ${ }^{27}$ (charge, polarity, volume, partition energy, hydrophobicity, proline signal (mutation to/from proline)).

The full dataset with 35 annotation features collected for all the CFTR missense variants can be found on GitHub (https://github.com/rychkova/CFTR-MetaPred).

\section{Machine learning model training}

To build the machine learning model, we utilized the statistical software program $\mathrm{R}$ with the library package caret ${ }^{28}$. To find the best performing algorithm, we tested several available methods: regularized logistic regression (GLM), regularized discriminant analysis (RDA), support vector machine (SVM), stochastic gradient boosting (tree boosting method) (GBM), and random forest (RF). The description of all the methods can be found in $\operatorname{ref}^{29}$. Of the 1,210 missense and initiator codon variants we annotated with 35 features, 295 unique variants had known clinical significance (161 pathogenic, 134 benign). We performed data preprocessing step by converting all the categorical features into numeric values, converting all the values into Z-scores, and imputing data with KNN method. It should be noted that a considerable amount of missing allele frequency data did not allow for a KNN imputation of this category, thus we used a dummy value of -1 for all the missing allele frequencies. We divided our dataset into training and testing sets with a ratio of 70/30. Five different models were built on the training set using five-fold cross validation for resampling, and the performance was measured on the test set. We have also estimated the performance of all the separate 35 features on the training set and compared it with the machine learning models.

\section{$\underline{\text { Results }}$}

Performance of all the five machine learning models we built and the 35 separate features can be found in Tables 3 and 4, respectively. Of the five models tested, RF showed the highest accuracy (77\%). Based on the AUC values, RF model outperformed all the other machine learning models $\left(\mathrm{AUC} \mathrm{CF}_{\mathrm{RF}}=\right.$ 85\%) (Fig. 2A), and it also improved over other popular tools, such as CADD (AUC CADD.RawScore $=$ 70\%), SIFT $\left(\mathrm{AUC}_{\mathrm{SIFT}}=63 \%\right)$, and PolyPhen-2 $\left(\mathrm{AUC}_{\mathrm{PolyPhen} 2}=60 \%\right)$ (see Fig. 2B and Table 4). Out of the 35 separate features, $\mathrm{AF}$ showed the best performance $\left(\mathrm{AUC}_{\mathrm{AF}}=73 \%\right)$ (Fig. 2B). $\mathrm{AF}$, Density, MutPred, POSE, and SIFT were selected as the most important features by the RF model (see Fig. 3 and Table S2). Interestingly, when looking at the features by their class (as defined in Table 2), features based on allele count (AF and Density) seem to be the most significant ones (Table S2), followed by 
sequence \& structure-based predictors (MutPred and POSE). SIFT was selected as the most significant one out of three sequence-based predictors we used (SIFT, PROVEAN, PANTHER). With regards to features derived from protein structure, protein topology (transmembrane helix, cytoplasmic or extracellular domain) and information about number of protein-protein interactions the residue participates in (PPI_score) showed higher importance, than other features calculated by DSSP for mutated protein models (HB_resi_change, HB_total_change, SAA_resi_change, SAA_total_change). From six physicochemical property features we derived from AAindex database (volume_change, polarity_change, partition_energy_change, hydrophobicity_change, charge_change, pro_signal), change in residue volume upon mutation seems to be the most important one.

To confirm our predictor's validity, we have also examined how predicted pathogenicity probability correlates with existing clinical and functional data. We used previously measured mean chloride conductance values $^{30}$ (Fig. 4A and Table S3) and sweat chloride data collected on patients at The Stanford CF Center (Fig. 4B and Table S4). The sweat chloride correlation analysis was restricted to patients heterozygous for p.F508del to reduce the variability due to different allele combinations. Both characteristics correlate well with the pathogenicity scores obtained using our RF classifier, with chloride conductance, which is a more direct measure of channel function, displaying the higher correlation coefficient $\left(\mathrm{R}^{2}=0.44\right)$. Mean chloride conductance values, as well as mean sweat chloride concentration values are listed in Tables S3 and S4, respectively.

Random forest pathogenicity probabilities along with predicted clinical significance categories for all the 1,210 missense CFTR variants can be found at GitHub (https://github.com/rychkova/CFTRMetaPred).

\section{$\underline{\text { Discussion }}$}

It has recently been recognized widely ${ }^{31-34}$ that computational predictors alone will not be able to reach satisfactory accuracy for direct use in the clinic, and both in vitro and in vivo functional studies are important to supplement the in silico predictions. Recognizing the importance of continuous-valued experimental quantitative measurements, rather than binary traits, Masica et al. ${ }^{31}$ extended their previously developed POSE method by including endophenotypic data from six clinical and functional assays. Their ePOSE (endoPhenotype-Optimized Sequence Ensemble) approach allows prediction of quantitative phenotypes associated with cystic fibrosis disease severity for missense variants in CFTR NBDs. Another study by Starita et al. ${ }^{32}$ explored the use of massively parallel experimental assays to 
measure the effect of nearly 2,000 missense substitutions in the RING domain of BRCA1 on its E3 ubiquitin ligase activity and its binding to the BARD1 RING domain. Model generated on the resulted scores was able to predict the capacities of full-length BRCAl variants, and outperformed widely used biological-effect prediction algorithms.

It should be noted that meta-predictor described here could easily be supplemented with functional data collected in high-throughput. In vitro functional measurements or even in vivo clinical phenotypes could be added as extra features during the model building step. With respect to CFTR protein, functional features like ion conductance, protein translation to the cell surface, and mRNA stability might be measured experimentally and added to the predictor for its overall performance improvement. Clinical phenotypes (like sweat chloride concentration, pancreatic sufficiency status, growth parameters, rate of first acquisition of Pseudomonas aeruginosa in the first year of life, and persistent colonization with $P$. aeruginosa) that are used in the clinic to help inform disease liability and penetrance of uncategorized mutations ${ }^{35,36}$, can be utilized as additional features in the prediction model as well. While our newly developed meta-predictor is not intended to uncover the molecular mechanisms of pathogenicity, it can help in prioritizing novel and rare genomic variations, identified through sequencing, for future functional studies. In addition, additional functional data may help to suggest potential cellular mechanism of the disease and allow for more accurate selection of specific therapies, as well as identify patients suitable for particular clinical trials.

The predictor described here could only be used to estimate a pathogenicity probability of missense variants, and its extension to other types of variants is somewhat less straightforward. The issue arises from the limited applicability of the predictors and structural features that we have utilized to build the model to classes of variants outside the missense category. In particular, only the methods PROVEAN and CADD can be applied to insertions and deletions, while nonsense, splicing, and synonymous mutations can be assessed by CADD only. To extract structural features, the structure of the mutated protein must be available, which is problematic for insertions and deletions that can cause large changes in structure. Moreover, the small set of TPs and TNs available for non-missense variant types limits our ability to train a similar meta-predictor, though allele frequency could in principle be used for any variant type. This again highlights the importance of additional functional measurements, which can be used alone or in combination with a few available computational features to establish the pathogenic status of all other types of variants. 
By combining multiple levels of knowledge about CFTR structure and function, and training the machine learning model on the set of known pathogenic and benign variants, we created a CFTRspecific pathogenicity predictor tool of higher accuracy, which we hope may aid in interpreting and prioritizing CFTR variants, and be further evaluated by functional studies. This model's predictions will be hosted on the ClinGen Consortium database, to make it easily available to other CF researchers and to demonstrate the feasibility of such an approach for a variety of Mendelian diseases. Overall, this report can be used as a description and model of the general strategy for developing a pathogenicity predictor of improved accuracy, so that feasibility of similar approaches may be evaluated for other genes.

\section{Description of Supplemental Data}

Supplemental Data include notes on data collection from Stanford internal resources (CF Center and MPL) and four tables.

\section{$\underline{\text { Acknowledgements }}$}

This work was supported by the ClinGen grant number U01 HG007436-01. AR would like to acknowledge additional support from The Dean's Postdoctoral Fellowship at the Stanford School of Medicine (2014-2015), and CEHG Postdoctoral Fellowship (2014-2015).

\section{Web Resources}

Complete list of 1,899 protein-coding CFTR variants with clinical significance, allele frequency and corresponding source dataset name, as well as 35 annotation features for 1,210 missense variants together with RF predictions, are available on the GitHub: https:/github.com/rychkova/CFTRMetaPred. 


\section{References}

1. Ng, P.C. (2003). SIFT: predicting amino acid changes that affect protein function. Nucleic Acids Res. $31,3812-3814$.

2. Adzhubei, I. a, Schmidt, S., Peshkin, L., Ramensky, V.E., Gerasimova, A., Bork, P., Kondrashov, A.S., and Sunyaev, S.R. (2010). A method and server for predicting damaging missense mutations. Nat. Methods 7, 248-249.

3. Thusberg, J., Olatubosun, A., and Vihinen, M. (2011). Performance of mutation pathogenicity prediction methods on missense variants. Hum. Mutat. 32, 358-368.

4. Li, B., Krishnan, V.G., Mort, M.E., Xin, F., Kamati, K.K., Cooper, D.N., Mooney, S.D., and Radivojac, P. (2009). Automated inference of molecular mechanisms of disease from amino acid substitutions. Bioinformatics 25, 2744-2750.

5. Masica, D.L., Sosnay, P.R., Cutting, G.R., and Karchin, R. (2012). Phenotype-Optimized Sequence Ensembles Substantially Improve Prediction of Disease-Causing Mutation in Cystic Fibrosis. Hum. Mutat.

6. Capriotti, E., Altman, R.B., and Bromberg, Y. (2013). Collective judgment predicts diseaseassociated single nucleotide variants. BMC Genomics 14 Suppl 3, S2.

7. Olatubosun, A., Väliaho, J., Härkönen, J., Thusberg, J., and Vihinen, M. (2012). PON-P: integrated predictor for pathogenicity of missense variants. Hum. Mutat. 33, 1166-1174.

8. Zhang, Z., Witham, S., Petukh, M., Moroy, G., Miteva, M., Ikeguchi, Y., and Alexov, E. (2013). A rational free energy-based approach to understanding and targeting disease-causing missense mutations. J. Am. Med. Inform. Assoc. 20, 643-651.

9. Serohijos, A.W.R., Aleksandrov, A.A., He, L., Cui, L., Dokholyan, N. V, and Riordan, J.R. (2008). Phenylalanine-508 mediates a cytoplasmic - membrane domain contact in the CFTR 3D structure crucial to assembly and channel function. Proc. Natl. Acad. Sci. 105, 3256-3261.

10. Dalton, J., Kalid, O., Schushan, M., Ben-Tal, N., and Villà-Freixa, J. (2012). New Model of Cystic Fibrosis Transmembrane Conductance Regulator Proposes Active Channel-like Conformation. J. Chem. Inf. Model. 52, 1842-1853.

11. Clinical and Functional Translation of CFTR (CFTR2). http://www.cftr2.org/

12. Database of Genotypes and Phenotypes (dbGaP). http://www.ncbi.nlm.nih.gov/gap

13. Exome Aggregation Consortium (ExAC). http://exac.broadinstitute.org

14. The Cystic Fibrosis Center at Stanford. http://med.stanford.edu/cfcenter.html

15. The Stanford Molecular Pathology Laboratory. http://moleculargenetics.stanford.edu/

16. Lefterova, M.I., Shen, P., Odegaard, J.I., Fung, E., Chiang, T., Peng, G., Davis, R.W., Wang, W., Kharrazi, M., Schrijver, I., et al. (2016). Next-Generation Molecular Testing of Newborn Dried Blood Spots for Cystic Fibrosis. J. Mol. Diagnostics 18, 267-282.

17. Choi, Y., Sims, G.E., Murphy, S., Miller, J.R., and Chan, A.P. (2012). Predicting the Functional Effect of Amino Acid Substitutions and Indels. PLoS One 7,.

18. Thomas, P.D., Campbell, M.J., Kejariwal, A., Mi, H., Karlak, B., Daverman, R., Diemer, K., Muruganujan, A., and Narechania, A. (2003). PANTHER: a library of protein families and subfamilies indexed by function. Genome Res. 13, 2129-2141. 
19. Kircher, M., Witten, D.M., Jain, P., O’Roak, B.J., Cooper, G.M., and Shendure, J. (2014). A general framework for estimating the relative pathogenicity of human genetic variants. Nat. Genet. 1-8.

20. Yin, S., Ding, F., and Dokholyan, N. V (2007). Eris : an automated estimator of protein. Nat. Methods 4, 466-467.

21. Dehouck, Y., Kwasigroch, J.M., Gilis, D., and Rooman, M. (2011). PoPMuSiC 2.1: a web server for the estimation of protein stability changes upon mutation and sequence optimality. BMC Bioinformatics 12,151 .

22. Schymkowitz, J., Borg, J., Stricher, F., Nys, R., Rousseau, F., and Serrano, L. (2005). The FoldX web server: An online force field. Nucleic Acids Res. 33, 382-388.

23. Consortium, T.U. (2014). UniProt: a hub for protein information. Nucleic Acids Res. 43, D204D212.

24. Warshel, A. (2013). Molaris-XG: Theoretical Background and Practical Examples.

25. Schrodinger LLC (2015). The PyMOL Molecular Graphics System, Version 1.8.

26. Touw, W.G., Baakman, C., Black, J., te Beek, T. a. H., Krieger, E., Joosten, R.P., and Vriend, G. (2014). A series of PDB-related databanks for everyday needs. Nucleic Acids Res. 43, D364-D368.

27. Kawashima, S., Pokarowski, P., Pokarowska, M., Kolinski, A., Katayama, T., and Kanehisa, M. (2008). AAindex : amino acid index database , progress report 2008. Nucleic Acids Res. 36, 202-205.

28. Kuhn, M. (2008). Building Predictive Models in R Using the caret Package. J. Stat. Softw. 28,.

29. Hastie, T., Tibshirani, R., and Friedman, J. (2009). The Elements of Statistical Learning: Data Mining, Inference, and Prediction (Springer).

30. Sosnay, P.R., Siklosi, K.R., Van Goor, F., Kaniecki, K., Yu, H., Sharma, N., Ramalho, A.S., Amaral, M.D., Dorfman, R., Zielenski, J., et al. (2013). Defining the disease liability of variants in the cystic fibrosis transmembrane conductance regulator gene. Nat. Genet. 45, 1160-1167.

31. Masica, D.L., Sosnay, P.R., Raraigh, K.S., Cutting, G.R., and Karchin, R. (2014). Missense variants in CFTR nucleotide-binding domains predict quantitative phenotypes associated with cystic fibrosis disease severity. Hum. Mol. Genet. 24, 1-10.

32. Starita, L.M., Young, D.L., Islam, M., Kitzman, J.O., Gullingsrud, J., Hause, R.J., Fowler, D.M., Parvin, J.D., Shendure, J., and Fields, S. (2015). Massively Parallel Functional Analysis of BRCA1 RING Domain Variants. Genetics 200, 413-422.

33. Ramalho, A.S., Clarke, L. a., Sousa, M., Felicio, V., Barreto, C., Lopes, C., and Amaral, M.D. (2015). Comparative ex vivo, in vitro and in silico analyses of a CFTR splicing mutation: Importance of functional studies to establish disease liability of mutations. J. Cyst. Fibros.

34. Masica, D.L., and Karchin, R. (2016). Towards Increasing the Clinical Relevance of In Silico Methods to Predict Pathogenic Missense Variants. PLOS Comput. Biol. 12, e1004725.

35. Raraigh, S., Keens, T.G., and Kharrazi, M. (2016). Benign and Deleterious Cystic Fibrosis Transmembrane Conductance Regulator Mutations Identified by Sequencing in Positive Cystic Fibrosis Newborn Screen Children from California. PLoS One 1-14.

36. Salinas, D.B., Sosnay, P.R., Azen, C., Young, S., Raraigh, K.S., Keens, T.G., and Kharrazi, M. (2015). Benign Outcome among Positive Cystic Fibrosis Newborn Screen Children with Non CFcausing Variants. J Cyst Fibros 14, 714-719. 
37. Guggino, W.B., and Stanton, B. a (2006). New insights into cystic fibrosis: molecular switches that regulate CFTR. Nat. Rev. Mol. Cell Biol. 7, 426-436.

38. Hallows, K.R., Raghuram, V., Kemp, B.E., Witters, L. a, and Foskett, J.K. (2000). Inhibition of cystic fibrosis transmembrane conductance regulator by novel interaction with the metabolic sensor AMP-activated protein kinase. J. Clin. Invest. 105, 1711-1721.

39. Cheng, J., Moyer, B.D., Milewski, M., Loffing, J., Ikeda, M., Mickle, J.E., Cutting, G.R., Li, M., Stanton, B. a., and Guggino, W.B. (2002). A golgi-associated PDZ domain protein modulates cystic fibrosis transmembrane regulator plasma membrane expression. J. Biol. Chem. 277, 3520-3529.

40. Jurkuvenaite, A., Varga, K., Nowotarski, K., Kirk, K.L., Sorscher, E.J., Li, Y., Clancy, J.P., Bebok, Z., and Collawn, J.F. (2006). Mutations in the amino terminus of the cystic fibrosis transmembrane conductance regulator enhance endocytosis. J. Biol. Chem. 281, 3329-3334. 
bioRxiv preprint doi: https://doi org/10.1101/115956; this version posted March 10, 2017. The copyright holder for this preprint (which was not certified by peer review) is the author/funder, who has granted bioRxiv a license to display the preprint in perpetuity. It is made available under aCC-BY 4.0 International license.

\section{$\underline{\text { Figures }}$}

Figure 1. Distribution of 1,899 collected protein coding CFTR variants by mutation type and clinical significance. (A) Missense variants represent the largest class of protein-coding variants in CFTR. (B) Majority of variants are classified as VUSs.

A

29

11

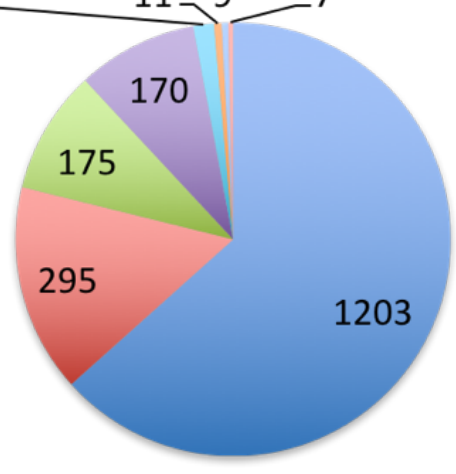

B

missense
frameshift
synonymous
stop gained
inframe deletion
splice region
inframe insertion
initiator codon

initiator codon

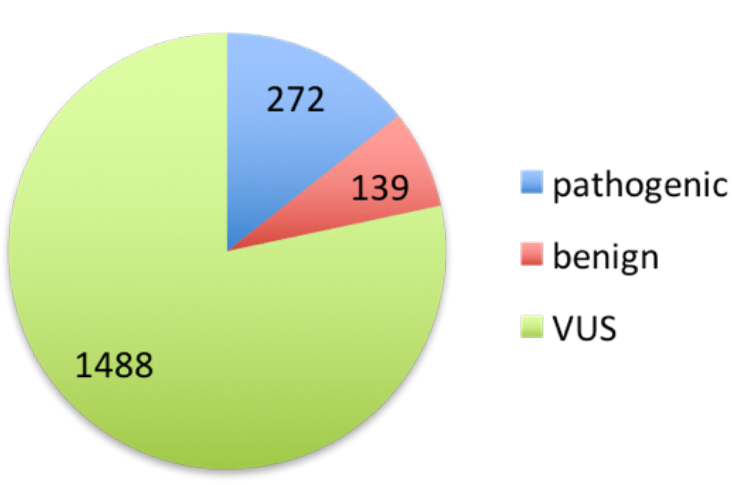


Figure 2. Performance comparison for ML algorithms tested and separate predictors used. (A) ROC curves for the five ML algorithms tested and their corresponding AUC values. Tree-based methods (GBM and RF) showed the highest performance, with the best $\mathrm{AUC}_{\mathrm{RF}}=0.85$. (B) $\mathrm{ROC}$ curves for the separate predictors used for training. Only five best predictors out of 35 shown for clarity. AF predictor showed the highest $\mathrm{AUC}_{\mathrm{AF}}=0.73$ in compare to other tools. AUC values for all 35 features are in Table 4.

A

ML methods

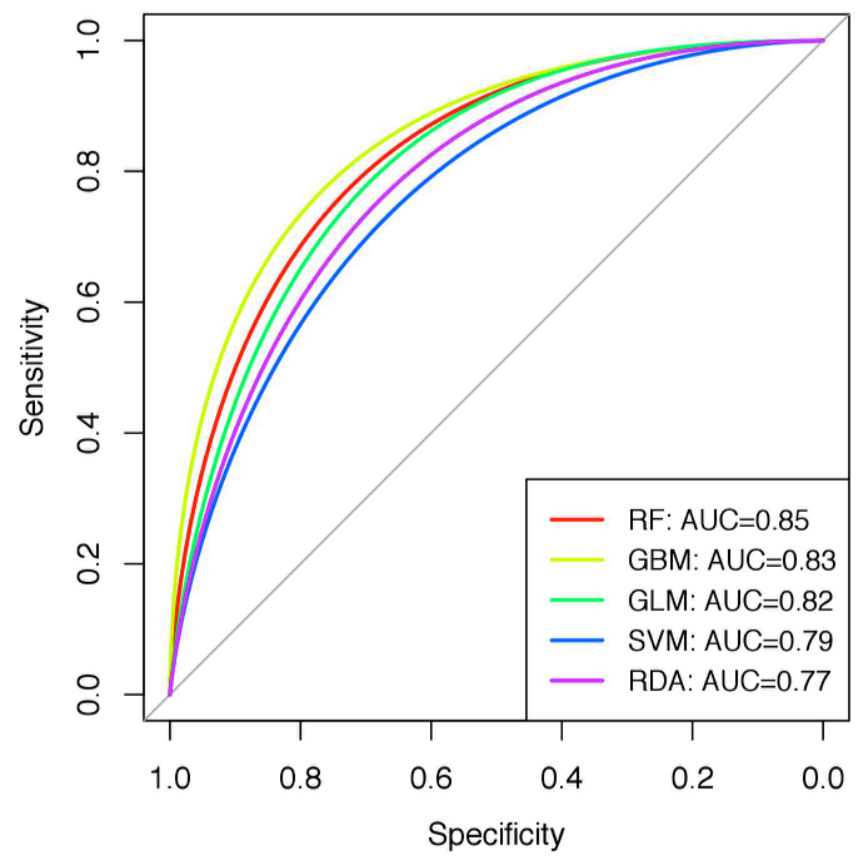

B

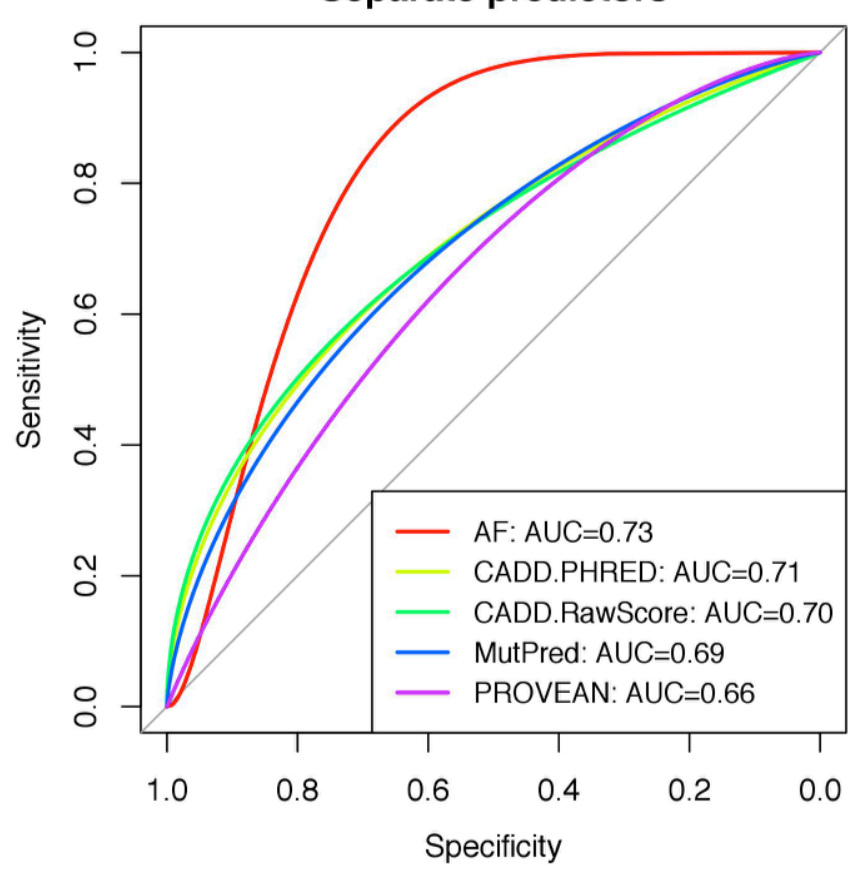


bioRxiv preprint doi: https://doi.org/10.1101/115956; this version posted March 10, 2017. The copyright holder for this preprint (which was not certified by peer review) is the author/funder, who has granted bioRxiv a license to display the preprint in perpetuity. It is made available under aCC-BY 4.0 International license.

Figure 3. Features importance based on the RF model. Only top 20 features are shown. Values are scored from 0 to 100. Data for all 35 features are in Table $\mathbf{S 2}$.

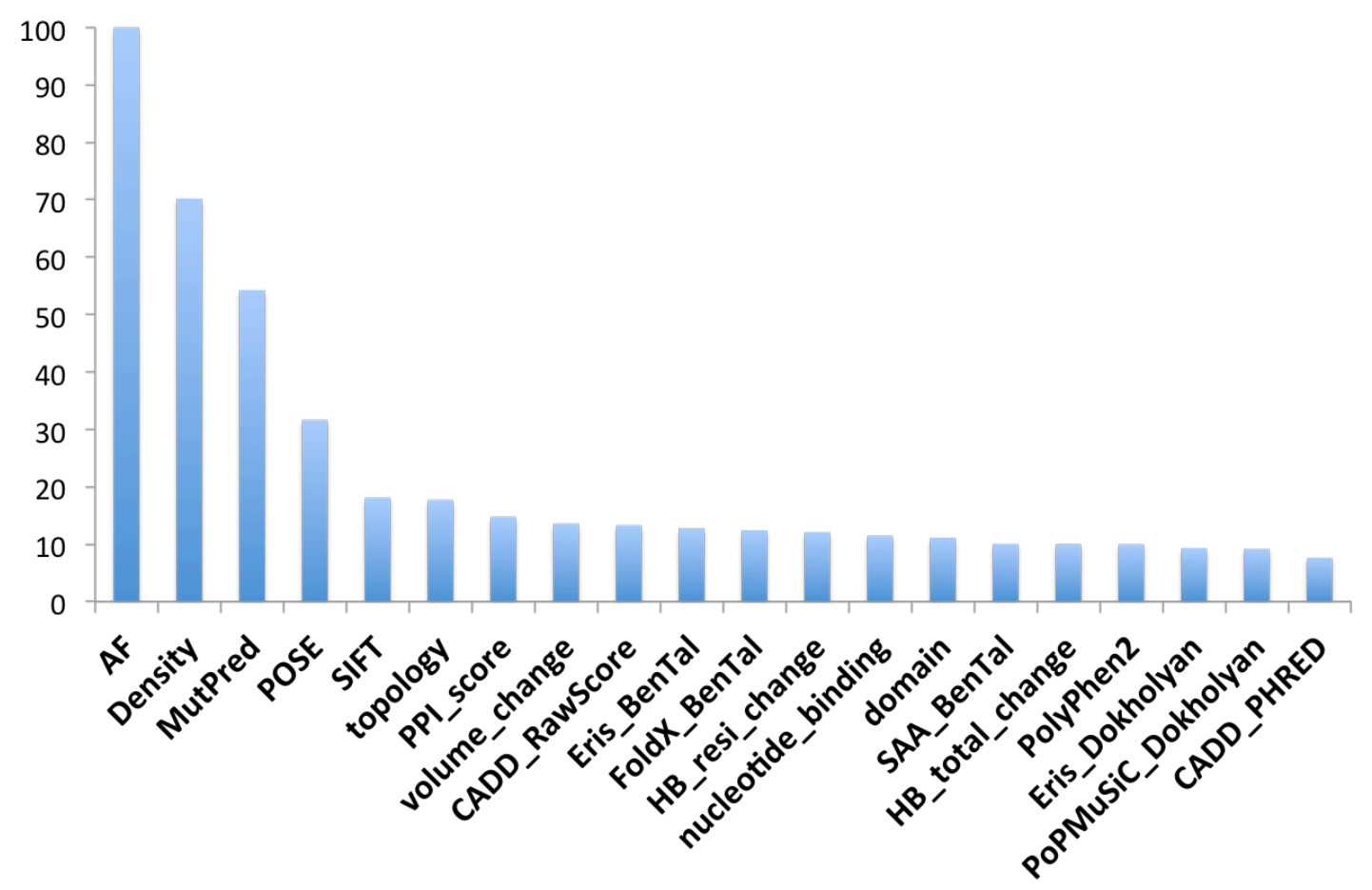


Figure 4. Correlation of pathogenicity prediction with experimental data. (A) Mean $\mathrm{Cl}^{-}$conductance values were taken from Sosnay et al. ${ }^{30}$. (B) Mean sweat $\mathrm{Cl}^{-}$conductance based on the data from The Stanford CF Center. Values for patients heterozygous for F508del mutation only were used. Pathogenicity probability is based on the predictions from RF model.

A

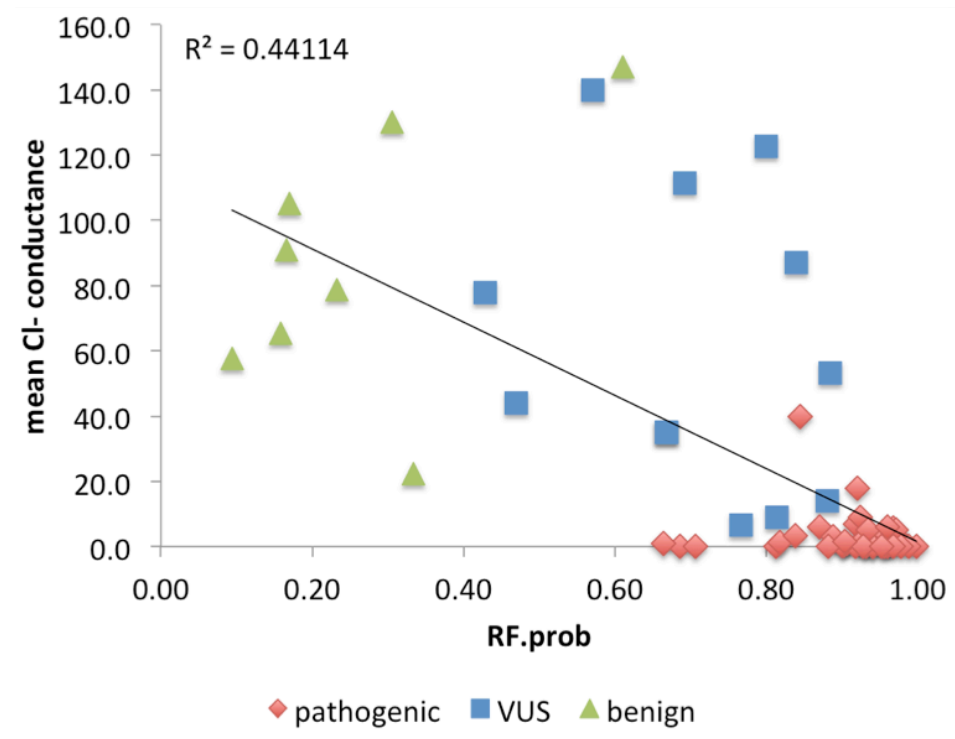

$\mathrm{B}$

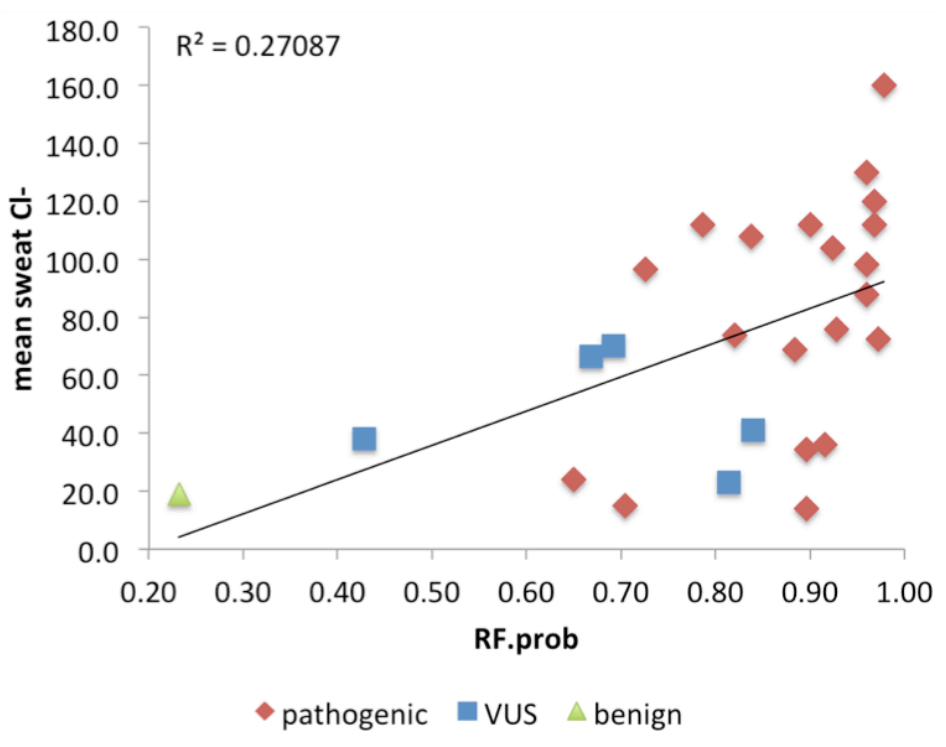




\section{$\underline{\text { Tables }}$}

Table 1. CFTR variants data collection

\begin{tabular}{|l|l|l|}
\hline datasource & $\begin{array}{l}\text { \# of } \\
\text { protein- } \\
\text { coding } \\
\text { variants }\end{array}$ & $\begin{array}{l}\text { \# of unique } \\
\text { variants for } \\
\text { this } \\
\text { datasource }\end{array}$ \\
\hline The Stanford Molecular Pathology Laboratory & 1267 & 726 \\
\hline The Exome Aggregation Consortium (ExAC) & 937 & 559 \\
\hline POSE $($ CFTR-specific pathogenicity prediction algorithm) & 240 & 37 \\
\hline The Clinical and Functional Translation for CFTR (CFTR2) & 171 & 4 \\
\hline The Stanford Cystic Fibrosis center & 80 & 5 \\
\hline The database of Genotypes and Phenotypes (dbGaP) & 53 & 9 \\
\hline Total: & $\mathbf{1 8 9 9}$ & \\
\hline
\end{tabular}

Variants data have been collected from six data sources, including external publicly available data and internal data from Stanford-affiliated laboratories. Total number of unique variants collected is 1,899 . *The Stanford MPL database that we used include variants identified during clinical CF testing at the lab, and laboratory-curated CFTR variants from various public datasources, including the CF Mutation Database, the CFTR2 database, National Center for Biotechnology Information's dbSNP, and Ensembl. 
Table 2. Features used for training machine learning algorithms

\begin{tabular}{|c|c|c|c|c|}
\hline \# & $\begin{array}{l}\text { feature } \\
\text { class }\end{array}$ & feature name & feature description & link/comment \\
\hline 1 & \multirow{3}{*}{$\begin{array}{l}\text { sequence- } \\
\text { based }\end{array}$} & PROVEAN & PROVEAN & http://provean.jcvi.org/ \\
\hline 2 & & SIFT & SIFT & http://sift.jcvi.org/ \\
\hline 3 & & PANTHER & PANTHER & $\begin{array}{l}\text { http://www.pantherdb.org/tools/csnpScor } \\
\text { eForm.jsp }\end{array}$ \\
\hline 4 & \multirow{4}{*}{$\begin{array}{l}\text { sequence \& } \\
\text { structure- } \\
\text { based }\end{array}$} & PolyPhen2 & PolyPhen-2 & http://genetics.bwh.harvard.edu/pph2/ \\
\hline 5 & & MutPred & MutPred & http://mutpred.mutdb.org/ \\
\hline $\begin{array}{l}6 \\
7\end{array}$ & & $\begin{array}{l}\text { CADD_RawScore, } \\
\text { CADD_PHRED }\end{array}$ & $\begin{array}{l}\text { CADD (RawScore and } \\
\text { PHRED) }\end{array}$ & $\underline{\text { http://cadd.gs.washington.edu/ }}$ \\
\hline 8 & & POSE & POSE & http://karchinlab.org/apps/appPose.html \\
\hline $\begin{array}{l}9 \\
10\end{array}$ & \multirow[t]{3}{*}{$\begin{array}{l}\text { stability } \\
\text { predictors }\end{array}$} & $\begin{array}{l}\text { PoPMuSiC_BenTal, } \\
\text { PoPMuSiC_Dokholyan }\end{array}$ & PoPMuSiC $^{*}$ & http://dezyme.com/ \\
\hline $\begin{array}{l}11 \\
12\end{array}$ & & $\begin{array}{l}\text { Eris_BenTal, } \\
\text { Eris_Dokholyan }\end{array}$ & Eris $^{*}$ & http://dokhlab.unc.edu/tools/eris/ \\
\hline $\begin{array}{l}13 \\
14\end{array}$ & & $\begin{array}{l}\text { FoldX_BenTal, } \\
\text { FoldX_Dokholyan }\end{array}$ & FoldX ${ }^{*}$ & http://foldx.crg.es/ \\
\hline 15 & \multirow{2}{*}{$\begin{array}{l}\text { additional } \\
\text { features } \\
\text { based on } \\
\text { allele count }\end{array}$} & $\mathrm{AF}$ & allele frequency & $\begin{array}{l}\text { based on allele count data from CFTR2, } \\
\text { dbGaP, ExAC, and Stanford CF Center }\end{array}$ \\
\hline 16 & & Density & probability density function & based on 1d kernel density estimate \\
\hline $\begin{array}{l}17 \\
18\end{array}$ & \multirow{12}{*}{$\begin{array}{l}\text { additional } \\
\text { features } \\
\text { derived } \\
\text { from } \\
\text { protein } \\
\text { structure }\end{array}$} & $\begin{array}{l}\text { SAA_BenTal, } \\
\text { SAA_Dokholyan }\end{array}$ & $\begin{array}{l}\text { solvent accessible area for } \\
\text { residue }^{*}\end{array}$ & \multirow[t]{5}{*}{ based on DSSP } \\
\hline 19 & & SAA_resi_change & $\begin{array}{l}\text { change in solvent accessible } \\
\text { area for residue }\end{array}$ & \\
\hline 20 & & SAA_total_change & $\begin{array}{l}\text { change in solvent accessible } \\
\text { area for protein }\end{array}$ & \\
\hline 21 & & HB_resi_change & $\begin{array}{l}\text { change in hydrogen bond } \\
\text { energy for residue }\end{array}$ & \\
\hline 22 & & HB_total_change & $\begin{array}{l}\text { change in hydrogen bong } \\
\text { energy for protein }\end{array}$ & \\
\hline 23 & & domain & protein domain & \multirow{7}{*}{$\begin{array}{l}\text { based on UniProt info and 3D protein } \\
\text { structure }\end{array}$} \\
\hline 24 & & nucleotide_binding & nucleotide binding pocket & \\
\hline 25 & & topology & topology & \\
\hline 26 & & modifications & posttranslational modification & \\
\hline 27 & & PPI_score & $\begin{array}{l}\text { involvement in protein- } \\
\text { protein interaction }\end{array}$ & \\
\hline 28 & & mem_cont_resi & $\begin{array}{l}\text { membrane contacting } \\
\text { residues }\end{array}$ & \\
\hline 29 & & ch_cont_resi & ion channel forming residues & \\
\hline 30 & \multirow{3}{*}{$\begin{array}{l}\text { physicoche } \\
\text { mical } \\
\text { properties }\end{array}$} & charge_change & change in charge & \multirow[t]{3}{*}{ based on AAindex } \\
\hline 31 & & polarity_change & change in polarity & \\
\hline 32 & & volume_change & change in volume & \\
\hline
\end{tabular}




\begin{tabular}{|l|l|l|l|}
\hline 33 & \multirow{2}{*}{34} & partition_energy_change & change in partition energy \\
\cline { 1 - 1 } 35 & hydrophobicity_change & change in hydrophobicity \\
\cline { 3 - 3 } & pro_signal & proline signal & \\
\hline
\end{tabular}

*PoPMuSiC, Eris, FoldX, as well as solvent accessible area for residues were calculated for two different CFTR structural models ${ }^{9,10}$, thus making 35 total annotation features. 
Table 3. Performance measures for the five machine learning algorithms tested

\begin{tabular}{|l|r|r|r|r|}
\hline method & accuracy & $\begin{array}{l}\text { sensitivity } \\
\text { (TPR=TP/P) }\end{array}$ & $\begin{array}{l}\text { lpecificity } \\
\text { (TNR=TN/N) }\end{array}$ & \multicolumn{1}{l|}{ AUC } \\
\hline RF (random forest) & 0.77 & 0.75 & 0.80 & 0.85 \\
\hline GBM (gradient boosting method) & 0.73 & 0.65 & 0.83 & 0.83 \\
\hline GLM (generalized linear model) & 0.55 & 1.00 & 0.00 & 0.82 \\
\hline SVM (support vector machine) & 0.68 & 0.71 & 0.65 & 0.79 \\
\hline $\begin{array}{l}\text { RDA (regularized discriminant } \\
\text { analysis) }\end{array}$ & 0.68 & 0.71 & 0.65 & 0.77 \\
\hline
\end{tabular}

TPR - true positive rate, TP - number of true positives, $\mathrm{P}$ - total number of positives (true positives + false positives), TNR - true negative rate, $\mathrm{TN}$ - number of true negatives, $\mathrm{N}$ - total number of negatives (true negatives + false negatives $)$. Accuracy $=(\mathrm{TP}+\mathrm{TN}) /(\mathrm{P}+\mathrm{N})$. 
Table 4. Performance measures for separate predictors

\begin{tabular}{|c|c|c|c|c|c|}
\hline feature & accuracy & $\begin{array}{l}\text { sensitivity } \\
(\mathrm{TPR}=\mathrm{TP} / \mathrm{P})\end{array}$ & $\begin{array}{l}\text { specificity } \\
(\mathrm{TNR}=\mathrm{TN} / \mathrm{N})\end{array}$ & AUC & $\begin{array}{l}\text { RF AUC } \\
\text { improvement } \\
, \%\end{array}$ \\
\hline $\mathrm{AF}$ & 0.73 & 0.69 & 0.78 & 0.73 & 11.9 \\
\hline CADD_PHRED & 0.64 & 0.75 & 0.50 & 0.71 & 14.1 \\
\hline CADD_RawScore & 0.63 & 0.60 & 0.65 & 0.70 & 15.0 \\
\hline MutPred & 0.60 & 0.83 & 0.33 & 0.69 & 15.5 \\
\hline PROVEAN & 0.65 & 0.69 & 0.60 & 0.66 & 19.0 \\
\hline PoPMuSiC_BenTal & 0.63 & 0.71 & 0.53 & 0.63 & 21.9 \\
\hline SIFT & 0.57 & 0.92 & 0.15 & 0.63 & 22.1 \\
\hline SAA_resi_change & 0.56 & 0.88 & 0.18 & 0.62 & 22.9 \\
\hline PANTHER & 0.60 & 0.73 & 0.45 & 0.62 & 23.0 \\
\hline PolyPhen2 & 0.57 & 0.83 & 0.25 & 0.60 & 25.1 \\
\hline SAA_Dokholyan & 0.61 & 0.69 & 0.53 & 0.59 & 26.2 \\
\hline FoldX_BenTal & 0.53 & 0.92 & 0.08 & 0.59 & 26.2 \\
\hline POSE & 0.52 & 0.67 & 0.35 & 0.58 & 26.8 \\
\hline charge_change & 0.53 & 0.85 & 0.15 & 0.58 & 27.3 \\
\hline SAA_total_change & 0.53 & 0.85 & 0.15 & 0.57 & 27.5 \\
\hline volume_change & 0.50 & 0.75 & 0.20 & 0.57 & 27.6 \\
\hline ch_contact_resi & 0.55 & 1.00 & 0.00 & 0.57 & 27.6 \\
\hline domain & 0.55 & 0.85 & 0.18 & 0.57 & 28.1 \\
\hline topology & 0.57 & 0.75 & 0.35 & 0.56 & 29.4 \\
\hline PPI_score & 0.55 & 1.00 & 0.00 & 0.55 & 29.9 \\
\hline FoldX_Dokholyan & 0.55 & 0.96 & 0.05 & 0.55 & 30.1 \\
\hline hydrophobicity_change & 0.53 & 0.83 & 0.18 & 0.55 & 30.1 \\
\hline PoPMuSiC_Dokholyan & 0.47 & 0.79 & 0.08 & 0.54 & 30.4 \\
\hline mem_contact_resi & 0.58 & 0.98 & 0.10 & 0.54 & 30.9 \\
\hline partition_energy_change & 0.50 & 0.90 & 0.03 & 0.54 & 31.3 \\
\hline pro_signal & 0.55 & 1.00 & 0.00 & 0.53 & 32.2 \\
\hline Eris_Dokholyan & 0.55 & 1.00 & 0.00 & 0.52 & 32.7 \\
\hline Density & 0.55 & 1.00 & 0.00 & 0.52 & 33.1 \\
\hline nucleotide_binding & 0.55 & 1.00 & 0.00 & 0.51 & 34.1 \\
\hline modifications & 0.55 & 1.00 & 0.00 & 0.51 & 34.3 \\
\hline polarity_change & 0.55 & 1.00 & 0.00 & 0.49 & 35.5 \\
\hline HB_resi_change & 0.55 & 0.98 & 0.03 & 0.48 & 36.6 \\
\hline HB_total_change & 0.55 & 0.98 & 0.03 & 0.48 & 36.6 \\
\hline Eris_BenTal & 0.52 & 0.92 & 0.05 & 0.48 & 36.6 \\
\hline
\end{tabular}


bioRxiv preprint doi: https://doi org/10.1101/115956; this version posted March 10,2017. The copyright holder for this preprint (which was not certified by peer review) is the author/funder, who has granted bioRxiv a license to display the preprint in perpetuity. It is made available under aCC-BY 4.0 International license.

\begin{tabular}{|l|r|r|r|r|r|}
\hline SAA_BenTal & 0.55 & 1.00 & 0.00 & 0.47 & 37.7 \\
\hline
\end{tabular}

Solvent accessible area (SAA), PoPMuSiC, Eris, and FoldX were calculated for two different CFTR structural models (BenTal ${ }^{10}$ and Dokholyan $\left.{ }^{9}\right)$. HB - hydrogen bond energy, PPI_score - number of protein-protein interactions this residue is known to participate in (see Table S1 for the PPI motifs details). 


\section{Supplemental Data}

On the data collection for the Stanford CF center

The Stanford CF Center provides state-of-the-art care for a patient population that comes from the San Francisco Bay area and beyond. The center currently cares for approximately 450 patients, including post-transplant CF patients. Our current standing protocol for clinical care includes detailed phenotypic characterization as well as complete CFTR mutation analysis in all the patients under follow up. Information of CFTR mutations identified and results of sweat chloride results is kept on a secure database managed by the Center.

On the data collection for Stanford MPL

The Stanford Molecular Pathology Laboratory provides clinical diagnostic testing for CF. The laboratory currently offers carrier screening (basic and expanded), diagnostic testing, and molecular testing associated with CF newborn screening for the State of California. This testing includes a 40 mutation screening panel for all newborns in the State of California who have a high initial IRT enzyme test result. A subset of these, namely those for whom only one mutation was identified through panel testing, receives further screening by direct DNA sequencing. 


\section{Supplemental Tables}

Table S1. Known CFTR motifs responsible for protein-protein interaction.

\begin{tabular}{|l|l|l|l|}
\hline PPI motif & motif description & $\begin{array}{l}\text { residues in } \\
\text { the motif }\end{array}$ & reference \\
\hline SNAREs_binding_motif & $\begin{array}{l}\text { Mediates membrane fusion and vesicle trafficking by } \\
\text { assembling into complexes that link vesicle-associated } \\
\text { SNAREs (v-SNAREs) with SNAREs on target membranes (t- } \\
\text { SNAREs). }\end{array}$ & $1-79$ & ref $^{37}$ \\
\hline AMPK_binding_motif & $\begin{array}{l}\text { A molecular switch that links ion transport to cellular } \\
\text { metabolism. AMPK is activated when the AMP/ATP ratio } \\
\text { increases. }\end{array}$ & $1420-1457$ & ref $^{38}$ \\
\hline PP2A_binding_motif & $\begin{array}{l}\text { A heterotrimeric protein phosphatase that interacts with and } \\
\text { dephosphorylates CFTR. }\end{array}$ & $1451-1476$ & ref \\
\hline PDZ_binding_motif & $\begin{array}{l}\text { Mediate protein-protein interactions by binding to short } \\
\text { peptide sequences that are most often in the C termini of the } \\
\text { target proteins. }\end{array}$ & $1477-1480$ & ref $^{39}$ \\
\hline endocytic_motif & $\begin{array}{l}\text { Regulates endocytosis by which extracellular material and } \\
\text { membrane-resident proteins are taken up by cells. }\end{array}$ & $\begin{array}{l}1424-1427, \\
1430-1431\end{array}$ & ref \\
\hline possible_endocytic_motif & $\begin{array}{l}{ }^{*} \text { Possible internalization signal suggested by R31C \& R31L } \\
\text { mutation experiments }\end{array}$ & $\begin{array}{l}28-31^{*}, \\
{ }^{* * *} \text { Possible internalization motif based on the structural } \\
\text { similarity to conventional internalization motifs in NBD2. }\end{array}$ & ref ${ }^{40}$ \\
\hline
\end{tabular}


Table S2. Importance for all 35 features on the scale 0-100.

\begin{tabular}{|c|c|c|}
\hline feature name & feature class & importance \\
\hline $\mathrm{AF}$ & derived from datasets & 100.0 \\
\hline Density & derived from datasets & 70.2 \\
\hline MutPred & sequence \& structure-based & 54.3 \\
\hline POSE & sequence \& structure-based & 31.6 \\
\hline SIFT & sequence-based & 18.1 \\
\hline topology & derived from protein structure & 17.7 \\
\hline PPI_score & derived from protein structure & 14.7 \\
\hline volume_change & physicochemical property & 13.6 \\
\hline CADD_RawScore & sequence \& structure-based & 13.3 \\
\hline Eris_BenTal & stability predictor & 12.8 \\
\hline FoldX_BenTal & stability predictor & 12.4 \\
\hline HB_resi_change & derived from protein structure & 12.1 \\
\hline nucleotide_binding & derived from protein structure & 11.4 \\
\hline domain & derived from protein structure & 11.1 \\
\hline SAA_BenTal & derived from protein structure & 10.0 \\
\hline HB_total_change & derived from protein structure & 10.0 \\
\hline PolyPhen2 & sequence \& structure-based & 9.9 \\
\hline Eris_Dokholyan & stability predictor & 9.3 \\
\hline PoPMuSiC_Dokholyan & stability predictor & 9.2 \\
\hline CADD_PHRED & sequence \& structure-based & 7.6 \\
\hline polarity_change & physicochemical property & 7.6 \\
\hline modifications & derived from protein structure & 7.0 \\
\hline PoPMuSiC_BenTal & stability predictor & 6.7 \\
\hline PROVEAN & sequence-based & 6.6 \\
\hline ch_contact_resi & derived from protein structure & 5.2 \\
\hline SAA_Dokholyan & derived from protein structure & 5.1 \\
\hline PANTHER & sequence-based & 4.4 \\
\hline SAA_total_change & derived from protein structure & 4.3 \\
\hline partition_energy_change & physicochemical property & 3.6 \\
\hline hydrophobicity_change & physicochemical property & 3.0 \\
\hline mem_contact_resi & derived from protein structure & 2.6 \\
\hline SAA_resi_change & derived from protein structure & 2.1 \\
\hline charge_change & physicochemical property & 1.5 \\
\hline pro_signal & physicochemical property & 0.5 \\
\hline FoldX_Dokholyan & stability predictor & 0.0 \\
\hline
\end{tabular}


Table S3. Experimental in vitro mean $\mathrm{Cl}^{-}$conductance measures for various CFTR missense variants.

\begin{tabular}{|c|c|c|c|c|}
\hline aachange & clin_sign & mean_Cl_cond & RF.prob & RF.pred \\
\hline G576A & benign & 147.0 & 0.61 & pathogenic \\
\hline $\mathrm{I} 148 \mathrm{~T}$ & benign & 91.1 & 0.17 & benign \\
\hline L997F & benign & 22.4 & 0.33 & benign \\
\hline R1162L & benign & 130.2 & 0.31 & benign \\
\hline $\mathrm{R} 31 \mathrm{C}$ & benign & 105.3 & 0.17 & benign \\
\hline R668C & benign & 57.6 & 0.09 & benign \\
\hline R75Q & benign & 65.4 & 0.16 & benign \\
\hline S1235R & benign & 78.7 & 0.23 & benign \\
\hline $\mathrm{A} 455 \mathrm{E}$ & pathogenic & 6.8 & 0.92 & pathogenic \\
\hline A559T & pathogenic & 0.0 & 1.00 & pathogenic \\
\hline $\mathrm{D} 110 \mathrm{H}$ & pathogenic & 9.1 & 0.92 & pathogenic \\
\hline D614G & pathogenic & 18.0 & 0.92 & pathogenic \\
\hline E92K & pathogenic & 0.2 & 0.90 & pathogenic \\
\hline G1244E & pathogenic & 1.0 & 0.96 & pathogenic \\
\hline G178R & pathogenic & 3.4 & 0.97 & pathogenic \\
\hline G551D & pathogenic & 1.3 & 0.96 & pathogenic \\
\hline G85E & pathogenic & 2.4 & 0.97 & pathogenic \\
\hline G970R & pathogenic & 2.8 & 0.95 & pathogenic \\
\hline $\mathrm{I} 1234 \mathrm{~V}$ & pathogenic & 39.9 & 0.84 & pathogenic \\
\hline I336K & pathogenic & 0.9 & 0.91 & pathogenic \\
\hline L1065P & pathogenic & 0.0 & 0.96 & pathogenic \\
\hline L1077P & pathogenic & 0.0 & 0.94 & pathogenic \\
\hline L206W & pathogenic & 5.7 & 0.97 & pathogenic \\
\hline L227R & pathogenic & 0.0 & 0.69 & pathogenic \\
\hline L467P & pathogenic & 0.0 & 0.92 & pathogenic \\
\hline L927P & pathogenic & 0.1 & 0.93 & pathogenic \\
\hline $\mathrm{M} 1101 \mathrm{~K}$ & pathogenic & 0.0 & 0.81 & pathogenic \\
\hline $\mathrm{M} 1 \mathrm{~V}$ & pathogenic & 0.7 & 0.93 & pathogenic \\
\hline N1303K & pathogenic & 0.5 & 0.90 & pathogenic \\
\hline P205S & pathogenic & 2.5 & 0.93 & pathogenic \\
\hline P67L & pathogenic & 6.0 & 0.87 & pathogenic \\
\hline R1066C & pathogenic & 0.0 & 0.71 & pathogenic \\
\hline $\mathrm{R} 1066 \mathrm{H}$ & pathogenic & 1.3 & 0.82 & pathogenic \\
\hline R117C & pathogenic & 3.4 & 0.84 & pathogenic \\
\hline R334W & pathogenic & 1.3 & 0.92 & pathogenic \\
\hline $\mathrm{R} 347 \mathrm{H}$ & pathogenic & 5.2 & 0.97 & pathogenic \\
\hline R347P & pathogenic & 0.0 & 0.94 & pathogenic \\
\hline R352Q & pathogenic & 3.1 & 0.89 & pathogenic \\
\hline R560K & pathogenic & 0.0 & 0.99 & pathogenic \\
\hline R560T & pathogenic & 0.1 & 0.98 & pathogenic \\
\hline
\end{tabular}




\begin{tabular}{|l|l|r|r|l|}
\hline S1251N & pathogenic & 5.2 & 0.93 & pathogenic \\
\hline S341P & pathogenic & 0.0 & 0.88 & pathogenic \\
\hline S492F & pathogenic & 0.0 & 0.93 & pathogenic \\
\hline S549N & pathogenic & 1.6 & 0.90 & pathogenic \\
\hline S549R & pathogenic & 0.1 & 0.96 & pathogenic \\
\hline S945L & pathogenic & 6.0 & 0.96 & pathogenic \\
\hline T338I & pathogenic & 1.0 & 0.66 & pathogenic \\
\hline V520F & pathogenic & 0.2 & 0.97 & pathogenic \\
\hline Y569D & pathogenic & 0.0 & 0.95 & pathogenic \\
\hline D1152H & VUS & 77.8 & 0.43 & benign \\
\hline D1270N & VUS & 53.2 & 0.88 & pathogenic \\
\hline D579G & VUS & 13.9 & 0.88 & pathogenic \\
\hline F1052V & VUS & 87.0 & 0.84 & pathogenic \\
\hline G1069R & VUS & 122.8 & 0.80 & pathogenic \\
\hline I1027T & VUS & 111.3 & 0.69 & pathogenic \\
\hline R1070W & VUS & 8.9 & 0.81 & pathogenic \\
\hline R117H & VUS & 35.0 & 0.67 & pathogenic \\
\hline R74W & VUS & 44.0 & 0.47 & benign \\
\hline S977F & VUS & 6.7 & 0.77 & pathogenic \\
\hline V754M & VUS & 140.0 & 0.57 & pathogenic \\
\hline
\end{tabular}

Clin_sign - clinical significance of variants, RF.prob and RF.pred - probability of pathogenicity and predicted pathogenicity class by $\mathrm{RF}$ model. Mean $\mathrm{Cl}^{-}$conductance values and clinical significance are from $\operatorname{ref}^{30}$. 
Table S4. Mean sweat $\mathrm{Cl}^{-}$concentration and $\mathrm{RF}$ probabilities for missense variants in $\mathrm{CF}$ patients of The Stanford CF Center heterozygous for F508del mutation.

\begin{tabular}{|l|l|r|r|l|}
\hline aachange & clin_sign & mean_sweat_CI & RF.prob & RF.pred \\
\hline S1235R & benign & 19.0 & 0.23 & benign \\
\hline D836Y & pathogenic & 15.0 & 0.70 & pathogenic \\
\hline E217G & pathogenic & 24.0 & 0.65 & pathogenic \\
\hline G194V & pathogenic & 112.0 & 0.79 & pathogenic \\
\hline G27R & pathogenic & 96.5 & 0.73 & pathogenic \\
\hline G551D & pathogenic & 98.1 & 0.96 & pathogenic \\
\hline G85E & pathogenic & 112.0 & 0.97 & pathogenic \\
\hline L1065P & pathogenic & 130.0 & 0.96 & pathogenic \\
\hline N1088D & pathogenic & 69.0 & 0.88 & pathogenic \\
\hline N1303K & pathogenic & 112.0 & 0.90 & pathogenic \\
\hline R117C & pathogenic & 108.0 & 0.84 & pathogenic \\
\hline R334W & pathogenic & 104.0 & 0.92 & pathogenic \\
\hline R347H & pathogenic & 72.5 & 0.97 & pathogenic \\
\hline R352W & pathogenic & 34.5 & 0.90 & pathogenic \\
\hline R560T & pathogenic & 160.0 & 0.98 & pathogenic \\
\hline S492F & pathogenic & 76.0 & 0.93 & pathogenic \\
\hline S945L & pathogenic & 88.0 & 0.96 & pathogenic \\
\hline V456A & pathogenic & 74.0 & 0.82 & pathogenic \\
\hline V520F & pathogenic & 120.0 & 0.97 & pathogenic \\
\hline V520I & pathogenic & 36.0 & 0.92 & pathogenic \\
\hline V562I & pathogenic & 14.0 & 0.90 & pathogenic \\
\hline D1152H & VUS & 38.0 & 0.43 & benign \\
\hline F1052V & VUS & 41.0 & 0.84 & pathogenic \\
\hline I1027T & VUS & 70.3 & 0.69 & pathogenic \\
\hline R1070W & VUS & 23.0 & 0.81 & pathogenic \\
\hline R117H & VUS & 66.6 & 0.67 & pathogenic \\
\hline
\end{tabular}

Clinical significance of variants is based on The Stanford CF Center data. 